\title{
Pediatric oncology for the general pediatrician
}

\author{
Introduction to the Educational series
}

\author{
Koenraad K. Norga
}

Received: 15 December 2010 / Accepted: 22 December 2010 /Published online: 11 January 2011

(C) Springer-Verlag 2011

\begin{abstract}
Since its establishment as a pediatric subspecialty oncology has become the near exclusive domain of specialized tertiary centers. This has greatly aided progress in the field with formation of exemplary and highly focused international consortia that have continued to streamline clinical research efforts. Recently, the rate of progress in terms of further improvements in overall outcome has paradoxically slowed. Novel strategies are therefore needed to assure continued advances. On the one hand, international and global consortia of subspecialists will continue to focus on the development of much needed improved therapies for those disease groups that have not yet seen their prognosis brighten, and on the other hand, there is a trend to invest in the development of joint-care initiatives that assure cost-effective access to standard therapy for all. This will require closer involvement of the general pediatrician in certain aspects of cancer care in its broadest sense. We hope the upcoming series will aid this process by highlighting selected topics in pediatric oncology that are likely to gain even more relevance for the general pediatrician in the years to come. They include: screening strategies for cancer predisposition syndromes; molecular diagnosis and risk-adjustment of therapy for acute leukemia in childhood; an overview of new agents in clinical development; and the impact of cancer treatment on fertility and available preservation options.
\end{abstract}

Keywords Pediatric oncology - General pediatrician · Joint care models

\section{K. K. Norga $(\bowtie)$}

Children's Hospital, University of Leuven,

Herestraat 49,

3000 Leuven, Belgium

e-mail: koen.norga@uz.kuleuven.be

\section{Pediatric oncology today and tomorrow}

The remarkable progress in the treatment of childhood cancer over the past 50 years is often cited as one of the major achievements of modern medicine. From a universally fatal group of diseases up until the 1950's, malignancies in childhood are now reaching 5-year survival rates of nearly $80 \%$ in the developed world [8]. Most of this progress has been the result, not so much of successful translation of novel mechanistic biological insights, but rather of continued incremental empirical augmentations of treatment protocols developed within collaborative clinical research consortia. After the introduction of multimodal treatment and combination chemotherapy, and later the establishment of bone marrow and stem cell transplantation, more recent notable milestones include the development of powerful antifungal drugs and the discovery of prognostic markers that provide a rational basis for treatment stratification. Only very recently has the introduction of targeted therapy as a result of insights, gained in the field of tumor biology, started to impact on the outcome of selected tumor types. Nevertheless, and paradoxically, the rate of improvement of outcome for children with cancer has altogether slowed over the past 10 years.

There are at least two reasons for this trend. First, major breakthroughs have already been achieved and the "lowhanging fruits" have now been picked. The challenges that remain are not surprisingly simply the hardest to tackle. Indeed, a number of tumor types remain for which prognosis has been disproportionally unaffected by all the advances made. These include, e.g., certain subtypes of acute lymphoblastic leukemia (ALL), a large fraction of acute myeloid leukemia (AML), nonresectable brain tumors, and metastatic sarcomas. Secondly, it is now clear that treatment intensification has reached its limits, in spite of greatly improved supportive care (including access to powerful antifungals, 
improved antivirals, and hematopoietic growth factors), and consequently, further progress will need to come from elsewhere.

Here are a few areas where we should hope to see significant progress in the years to come.

The recent plethora of genetic data revealing the molecular architecture of different tumor types and stages will likely yield more molecular markers that can be used to stratify patients more accurately [6]. This will allow careful treatment de-intensification in selected subgroups to reduce immediate and late toxicity, while maintaining excellent tumor control, and at the same time, correct identification of those patients most likely to benefit from maximally intensive treatment schedules. These molecular data may also yield novel therapeutic targets for the development of new cancer drugs over the following decades. Technological and scientific breakthroughs will surely be needed to yield radically new treatment modalities for those tumor types that fail to respond to current approaches. Clinical development of any such novel agents or modalities will require the use of creative and innovative trial designs that will allow efficient detection of meaningful differences between low numbers of treated patients, which will be, more than before, geographically dispersed and molecularly heterogeneous [3].

Another area of potential progress pertains to late effects of treatment [7]. Better stratification will prevent late effects from occurring in patients with reduced-risk tumors. Molecular markers may allow early identification of those at risk for developing late toxicities. As we learn more about their pathophysiology, we will also get better at diagnosing, and eventually, at treating these iatrogenic disorders. This will have some impact on the long-term survival of children with cancer, but it may significantly affect their quality of life. As the number of childhood cancer survivors continues to grow, general pediatricians, and later, adult primary care physicians will have an increasing role in recognizing these late effects.

While progress toward event-free and long-term survival in childhood cancer has been remarkable in the developed world, the same has unfortunately not yet been true for the developing countries. One of the major challenges for pediatric oncologists worldwide is to now support the dispersal of the great successes in this field to the rest of the world [1]. This will require the design of maximally costeffective and safe treatment protocols, which, moreover, will also benefit the developed world in the context of budgetary restrictions in health care.

\section{Four upcoming contributions: an update for the general pediatrician}

Pediatric oncology has been, perhaps more than other subspecialties, the exclusive domain of specialized tertiary centers. The practice of referring patients to specialized centers for diagnosis, treatment and even long-term followup has certainly facilitated clinical research in this field. But with increasing consensus over standard treatment protocols for certain more common and highly tractable cancer types, such as reduced-risk ALL and early-stage solid tumors, pediatric oncologists are now beginning to develop joint-care models involving general pediatricians. To assure the best possible comprehensive care for our patients, the referring general pediatrician is increasingly a vital team member at various phases in the disease process, from detection of children at risk over administration of selected oncologic treatments to management of late effects. In the upcoming series, we have invited four reknowned experts to share their insights on selected topics, which we believe are relevant for general pediatricians with an interest in this area.

Kim E. Nichols is Associate Professor of Pediatrics at the University of Pennsylvania, and she directs the Pediatric Hereditary Cancer Predisposition Program at the Children's Hospital of Philadelphia. Together with her colleagues, Drs. Teplick, Kowalsky, and Biegel, she discusses with great clarity the issue of screening strategies for cancer predisposition syndromes [9]. Using selected examples along the spectrum of disorders for which screening guidelines are more or respectively less well established, they offer practical recommendations for the general pediatrician, who is typically the first one to suspect the presence of a predisposition syndrome.

Judy Margolin is Associate Professor of Pediatrics at Baylor College of Medicine and an active member on the Leukemia/Lymphoma team at the Texas Children's Cancer Center. In her contribution, she provides an overview of the current state-of-the-art on the molecular diagnosis of acute leukemia in childhood, and she introduces the general pediatrician to the concept of risk-adjusted therapy [5]. This strategy has been best established for acute lymphoblastic leukemia, which is likely to serve as a paradigm for other malignancies. Risk-adjusted therapy brings about novel opportunities for joint care models with participation of the child's referring pediatrician in treatment and followup for selected diseases, such as reduced-risk ALL.

Terzah Horton is Assistant Professor of Pediatrics at Baylor College of Medicine and an active member of the Developmental Therapeutics and Clinical Pharmacology Program at Texas Children's Cancer Center, directed by Stacey Berg (who is co-author on the article for this series). The Texas Children's Pediatric Pharmacology Research Unit is a pioneer in a field, which is now receiving more attention from industry, thanks to the new regulations at FDA and EMA that mandate testing of novel drugs in children by drug companies in exchange for patent extension. The authors give a structured overview of the 
new agents currently under clinical development in pediatric oncology [4]. While general pediatricians are unlikely to prescribe these drugs, it is relevant for them to be familiar with the dynamics of the clinical development process and to be aware of novel types of agents that may well transform the practice of pediatric oncology. Despite all the progress in molecular oncology, the number of new agents in clinical development for pediatric oncology remains rather limited.

Jill P. Ginsberg is Associate Professor of Pediatrics at the Center for Childhood Cancer Research and the Division of Oncology at the Children's Hospital of Philadelphia, University of Pennsylvania, where she directs the Cancer Survivorship Program. She has contributed a superb overview of a critical topic that has received significant attention recently in the field of late effects, i.e., the impact of cancer treatment on fertility and potential fertility preservation options available to our patients [2]. Dr. Ginsberg's article underscores the importance of considerating this issue at the time of diagnosis. For the referring pediatrician, it is important to have a realistic idea of risks and preservation options so that he/ she can effectively help to support patients and their families throughout the hectic phase of cancer diagnosis and therapy initiation.

We hope that these contributions will spark your interest in the fascinating field of pediatric oncology. Despite tremendous progress over the past decades continued challenges are awaiting that need the valued participation of general pediatricians in the comprehensive care for the patients that we share.

\section{References}

1. Eden T, Pui CH, Schrappe M, Tognoni G et al (2004) All children have a right to full access to treatment for cancer. Lancet 364 (9440):1121-1122

2. Ginsberg JP (2011) The effect of cancer on fertility and potential fertility preservation options for pediatric patients. Eur J Pediatr doi:10.1007/s00431-010-1359-4

3. Henschel AD, Rothenberger LG, Boos J (2010) Randomized clinical trials in children-ethical and methodological issues. Curr Pharm Des 16(22):2407-2415

4. Horton TM, Berg SL (2011) The development of new therapies for pediatric oncology.Eur J Pediatr doi:10.1007/s00431-010-1374-5

5. Margolin J (2011) Molecular diagnosis and risk-adjusted therapy in pediatric hematologic malignancies: a primer for pediatricians. Eur $\mathrm{J}$ Pediatr (in press)

6. Meyerson M, Gabriel S, Getz G (2010) Advances in understanding cancer genomes through second-generation sequencing. Nat Rev Genet 11(10):685-696

7. Robison LL, Armstrong GT, Boice JD, Chow EJ et al (2009) The Childhood Cancer Survivor Study: a National Cancer Institutesupported resource for outcome and intervention research. J Clin Oncol 27(14):2308-2318

8. Smith MA, Seibel NL, Altekruse SF, Ries LA, Melbert DL, O'Leary M, Smith FO, Reaman GH (2010) Outcomes for children and adolescents with cancer: challenges for the twenty-first century. J Clin Oncol 28(15):2625-2634

9. Teplick A, Kowalski M, Biegel JA, Nichols KE (2011) Screening in cancer predisposition syndromes: guidelines for the general pediatrician. Eur J Pediatr doi:10.1007/s00431-010-1377-2 